\title{
Civilisations
}

Revue internationale d'anthropologie et de sciences

humaines

$57 \mid 2008$

Tourisme, mobilités et altérités contemporaines

\section{« Wagah Border »}

Mise en tourisme d'un rituel nationaliste à la frontière indo-pakistanaise

\section{David Goeury}

\section{(2) OpenEdition}

1 Journals

Édition électronique

URL : http://journals.openedition.org/civilisations/1225

DOI : 10.4000/civilisations. 1225

ISSN : 2032-0442

Éditeur

Institut de sociologie de l'Université Libre de Bruxelles

\section{Édition imprimée}

Date de publication : 29 décembre 2008

Pagination : 139-154

ISBN : 2-87263-022-8

ISSN : 0009-8140

\section{Référence électronique}

David Goeury, « «Wagah Border » », Civilisations [En ligne], 57 | 2008, mis en ligne le 29 décembre

2011, consulté le 01 mai 2019. URL : http://journals.openedition.org/civilisations/1225 ; DOI :

10.4000/civilisations. 1225

(c) Tous droits réservés 


\title{
2. Frontières
}

\author{
"Wagah Border" \\ Mise en tourisme d'un rituel nationaliste à la frontière indo-pakistanaise
}

\author{
David GOEURY
}

Résumé : Sur $2912 \mathrm{~km}$ de la line of control qui sert de frontière entre l'Union indienne et le Pakistan depuis 1949, Wagah Border est l'un des deux Joint Check Point qui autorise le passage régulier d'individus et de marchandises. Chaque soir, les deux forces de sécurité, indienne et pakistanaise, ferment la frontière en sonnant la retraite et en descendant les deux drapeaux. Depuis 2000, le département du Tourisme du Punjab indien, puis les autorités pakistanaises, ont décidé de promouvoir cette cérémonie comme une destination touristique nationale. Des gradins ont été construits et la frontière est devenue le lieu d'une immense communion nationaliste. Le long prologue avant la cérémonie militaire se transforme en théatre où la foule y joue un rôle actif. La description de ce rituel collectif de plus en plus organisé (musique, chants, danses, courses de drapeaux) dans un espace aussi spécifique permet de réfléchir sur ce que représente la frontière comme enveloppe de la nation. En fait, si ce rituel donne à voir à certains égards une confrontation, sa mise en tourisme le pacifie, et contribue à transformer l'événement en la première étape d'un projet plus vaste de réconciliation et d'ouverture.

Mots-clefs : Inde, Pakistan, tourisme, nationalisme, frontière.

\begin{abstract}
Along the $2912 \mathrm{~km}$ of the 1949's Indian-Pakistan border, Wagah is one of the two Joint Check Point where people and goods are allowed to cross the border. Every evening, the Indian Border Security Force and the Pakistani Rangers ceremonially close the border gates and pull down the two national flags. Since 2000, the Indian Punjabi tourism office and, later on, the Pakistani administration have decided to promote the ceremony as a touristic destination. They built tiers and the border became the place of an intense nationalist communion. The long prologue before the military show turns into a theatre stage where the crowd contributes to the performance. The description of this more and more organized, collective ritual (music, songs, dances, flags'race) in this specific place allows to rethink the place of the borders in the making of a nation. In fact, if this ritual may somehow look like a confrontation, tourism pacifies it, and may actually transform the event in the first step of a mutual reconciliation and opening process.
\end{abstract}

Key words: India, Pakistan, tourism, nationalism, border. 


\section{Introduction}

$\mathrm{S}$ Point, avec Khokhrapar dans le Sindh, permettant le passage terrestre entre l'Union indienne et le Pakistan. Jusqu'en novembre 2007, la journée s'y écoulait lentement avec le passage de quelques véhicules, de quelques dizaines d'individus (les jours du bus Delhi-Lahore) et le mouvement des coolies pour décharger un éventuel camion de fruits et de légumes. Wagah semblait relier deux extrémités de deux mondes lointains qui entretiennent des relations ténues et suspicieuses. Pourtant, alors que le jour décline, que les derniers fonctionnaires quittent leur poste pour s'en retourner vers Amritsar pour les Indiens, vers Lahore pour les Pakistanais, la route s'enfièvre. Des dizaines d'échoppes ouvrent, autant de marchands ambulants s'installent sur le bord de la route, et surtout des centaines de véhicules, auto-rikshaws, taxis, bus, tentent de se garer bruyamment sur des bas-côtés non stabilisés, sur les conseils de gardiens de parking improvisés. La foule - plusieurs milliers en Inde, plusieurs centaines au Pakistan - s'agglutine devant la barrière des douanes. À $17 \mathrm{~h}$, à leur ouverture, elle entre en marche soutenue jusqu'au point « $0 »$ qui marque la frontière entre le Pakistan et l'Union indienne. Elle dépasse les administrations et se masse derrière une arche fermée d'un mince cordon tenu par les immenses soldats indiens, ou les puissants Rangers pakistanais. Vers 18h, enfin, les soldats font glisser la masse d'impatients le long de barrières métalliques qui longent la frontière, les Pakistanais les premiers puis les Indiens. Chacun prend une photo devant le petit bloc de béton qui marque le point « $0 »$ originel, puis renouvelle la prise de vue devant les deux portes (à vantaux pour l'Inde, coulissante pour le Pakistan) qui séparent les deux territoires avant de prendre place dans de vastes gradins roses en « $\mathrm{L}$ » pour l'Inde, et blancs en demi-cercle pour le Pakistan. L'attente n'est pas silencieuse, bien au contraire, les chants, les musiques et les danses s'enchaînent jusqu'à la tombée du jour. Puis, l'officier sonne la « retraite» avant la descente des drapeaux. Les gardes indiens à l'Est, les Rangers pakistanais à l'Ouest marchent d'un pas décidé vers les portes closes à grand renfort de gestes guerriers. Ils ouvrent les portes sous les acclamations. Commence alors une étrange parade où Pakistanais et Indiens, de façon coordonnée, grimacent, gesticulent, s'intimident, avant de faire glisser les deux drapeaux lentement et simultanément. Jamais l'un ne doit être plus haut que l'autre. Une fois les drapeaux pliés, les deux officiers se saluent avant de claquer les portes qui resteront closes toute la nuit. Alors, la foule quitte les gradins, non sans venir prendre quelques ultimes photographies avec les héros de la cérémonie.

Pour de nombreux auteurs (Nayar 2003; Tikekar 2004; Van Schendel 2007), la popularité de Wagah Border et de sa cérémonie guerrière serait le symbole de la violence perpétuelle entre Indiens et Pakistanais. Cependant, l'enquête de terrain et la rencontre des acteurs qui vivent la cérémonie (forces de sécurités frontalières, administrations locales, professionnels du tourisme, simples visiteurs) révèlent des logiques diverses, beaucoup plus complexes. Dès lors, quelle est l'impact de la mise en tourisme d'un lieu qui porte en lui la mémoire collective d'un conflit (Halbwachs 1950 : 209) ? Cette mise en tourisme serait-elle la preuve de l'existence de deux nations irréconciliables, prêtes à en découdre au moindre incident ? Ou participerait-elle d'un processus de pacification? Pour y répondre, nous voudrions confronter les multiples interprétations autour de la symbolique de la cérémonie à l'appropriation effective de la cérémonie par les visiteurs. 
Dès lors, la ritualisation de l'entrée des touristes dans les gradins et la théâtralisation de la fermeture de la frontière, peuvent être présentées comme une reconnaissance des deux nations, où chaque citoyen viendrait faire corps avec sa nation et attester de la partition effective entre les deux États. De ce fait, il n'y aurait pas d'incompatibilité entre le fait d'exalter des valeurs nationalistes et le processus de normalisation des relations bilatérales entre les deux États.

\section{Wagah Border, la naissance d'une destination sur le front indo- pakistanais}

C'est en 2000 que l'office du tourisme du Punjab indien décide de transformer Wagah en attraction touristique. La médiatisation de l'ouverture de la ligne de bus LahoreDelhi en février 1999 et l'engouement pour les cérémonies militaires incitent ses cadres à penser que Wagah pourrait devenir une destination à même d'allonger la durée des séjours touristiques à Amritsar. L'office du tourisme avec l'accord de la Border Security Force décide de construire des gradins à proximité du « point 0 ». Par réaction, l'autorité pakistanaise fait construire la tribune de Baab-e-Azadi (la porte de la liberté), inaugurée en août 2001, en hommage aux milliers de Pakistanais morts lors de la partition.

En quelques années, la cérémonie de Wagah est devenue une destination incontournable du tourisme national. Les jours de fête nationale, l'office du tourisme d'Amritsar estime que plus de 12000 visiteurs indiens assistent à la cérémonie. Désormais, chaque année, près de deux millions de touristes viendraient à la frontière ${ }^{1}$. La réussite d'un tel lieu et l'engouement populaire qu'ils suscitent dans toute l'Inde et dans tout le Pakistan est le fruit d'une accumulation de phénomènes (Equipe MIT 2005 : 8-9).

Wagah serait noyée dans l'immensité de la plaine punjabie, entrecoupée des seuls canaux d'irrigation, si la distinction entre les deux Punjab, l'État indien et la province pakistanaise, n'était pas marquée spatialement par une double clôture barbelée électrifiée (Foucher 1991 : 299). Née dans la douleur de la partition, la frontière Indo-pakistanaise reste une zone de conflits constants. Du Gujarat au Siachen, elle reste très fortement militarisée et le lieu d'affrontements plus ou moins violents. La dernière guerre dite « de Kargil » qui a eu lieu 1999 n'a pas mis fin aux multiples accrochages. La frontière, surtout le long du Cachemire, reste un lieu où meurent régulièrement des soldats ${ }^{2}$.

Or, Wagah est unique, c'est l'un des deux seuls Joint Check Point ${ }^{3}$ ouverts à la circulation des nationaux et des internationaux. La frontière est sous l'autorité des forces de sécurité de $16 \mathrm{~h}$ à $8 \mathrm{~h}$, des Custom Department et Immigration Department de $8 \mathrm{~h}$ à $16 \mathrm{~h}$. Jusqu'en novembre 2007, la circulation journalière restait très faible avec moins de cinq véhicules et à peine 75 personnes composées pour moitié d'Indiens et de Pakistanais, et

1. Ces chiffres ne sont que des estimations, la cérémonie étant gratuite, les visiteurs ne sont pas systématiquement décomptés. Les officiers de la Border Security Force estiment la fréquentation au remplissage des gradins.

2. Pour en prendre la mesure, il suffit de se rendre au monument aux morts de Chandigarh qui rend hommage à tous les soldats punjabis tombés au combat. Cette double spirale de béton sur laquelle sont accrochées des plaques de marbre noir gravées, se termine par plusieurs mètres d'emplacements vides prévus pour accueillir tous les noms de ceux qui vont mourir lors des accrochages frontaliers futurs.

3. Les Joint Check Points ont été créés en 1949. Ce sont les points où les deux forces de sécurités indienne et pakistanaise sont en contact. 
pour moitié de touristes étrangers. En 2007, ce point de passage ne voyait transiter que 5\% des échanges entre l'Inde et la Pakistan, soit moins de deux millions de roupies par an ${ }^{4}$. Les individus doivent être munis de visas obtenus à New Delhi ou à Islamabad, souvent après une attente de plusieurs jours. Le passage des véhicules indiens ou pakistanais était interdit, mis à part le bus Delhi/Lahore, qui passe quatre fois par semaine (deux aller-retour), et ceux des pèlerins sikhs indiens se rendant Nankana Sahib ${ }^{5}$. Avant le $1^{\text {er }}$ novembre 2007, les camions étaient obligés de décharger leur marchandise, composée de fruits et de légumes, qui étaient portées par des dizaines de coolies sur les centaines de mètres séparant les deux bureaux des douanes indiennes et pakistanaises. L'essentiel du commerce entre Amritsar et Lahore se fait via les ports de Bombay et de Karachi ${ }^{6}$.

La faiblesse numérique des passages de personnes et de marchandises ne doit pas cacher la forte résonance symbolique de Wagah Border. Point de passage terrestre, il incarne les relations entre le Pakistan et l'Union indienne. Il est régulièrement le lieu de gestes politiques bilatéraux entre les deux gouvernements. Ces actions sont alors largement couvertes par les médias. Wagah est un terme qui évoque, pour les nationaux, les relations entre l'Inde et le Pakistan?.

Ainsi, c'est le lieu de l'organisation des échanges de prisonniers indiens et pakistanais, civils ou militaires, capturés pour violation du territoire. Le 14 août 2007, l'Inde a libéré 72 Pakistanais, dont 48 pêcheurs arraisonnés dans les eaux territoriales indiennes, alors que le Pakistan renvoyait 134 Indiens, dont 100 pêcheurs. L'échange avait été préparé plusieurs semaines à l'avance, pour symboliquement correspondre au $60^{\mathrm{e}}$ anniversaire de l'indépendance des deux États ${ }^{8}$.

Mais c'est surtout en février 1999 que Wagah a pris une nouvelle dimension. Le Premier ministre indien Atal Bihari Vajpayee décide de se rendre au Pakistan via « Wagah Border », en bus, pour inaugurer la ligne Delhi-Lahore, avec 22 parlementaires indiens. Ce trajet terrestre, qui est aujourd'hui ouvert à la population, est le symbole d'une tentative de normalisation des relations indo-pakistanaises. Entre Amritsar à Wagah, les Indiens s'étaient massés pour l'occasion le long de la route formant une immense haie d'honneur de près de $35 \mathrm{~km}$. Le bus transportant le Premier ministre s'était arrêté à la frontière, puis était reparti alors que les Pakistanais se sont regroupés tout le long des $26 \mathrm{~km}$ qui séparent Wagah de Lahore. Pour l'éditorialiste et parlementaire indien Kuldip Nayar, cet événement a montré l'union possible des deux États autour d'une même chaîne humaine

4. Le volume des échanges en 2006 était de 869 millions de dollars, in Taneja N., Trade Possibilities and Non-Tariff Barriers to Indo-Pak Trade, New-Delhi: ICRIER, 2007, 47 p. Consultable en ligne. http://www.icrier.org/publication/WorkingPaperno200.pdf

5. Cette ville du Punjab pakistanais est le lieu de naissance de Guru Nanak, prophète de la religion sikhe.

6. Les entrepreneurs punjabis indiens spécialisés dans l'industrie mécanique regrettent de ne pas pouvoir écouler leurs produits directement sur le marché du Punjab pakistanais, pourtant fortement demandeur.

7. Cette dimension symbolique se retrouve dans les titres de livres.

8. "India to free 72 Pakistani prisoners", Daily Times, Tuesday, August 14, 2007. 
continue de $51 \mathrm{~km}$ (Nayar 2003 : 210). Le voyage s'était achevé le 20 février 1999 par la déclaration de Lahore qui entend rétablir des liens d'amitiés basés sur le commerce et la dénucléarisation de la région?.

Wagah est donc un lieu de la géographie mentale nationale, qu'elle soit pakistanaise ou indienne. D'ailleurs, alors que Wagah est le nom du village pakistanais, il supplante dans les deux États celui d'Attari, qui est le nom du village indien. Cette résonance est suffisante pour en faire un lieu de tourisme de frontière, où l'on vient à la fois commémorer une histoire, voir l'iconographie de la frontière avec ses fortifications, ses administrations, ses drapeaux et ses soldats, et observer un territoire à la fois proche et inaccessible (Gelbman 2008 : 201-202). L'attractivité du lieu est renforcée par la présence de forces paramilitaires qui, chaque soir, sonnent la retraite et organisent la descente simultanée des drapeaux. Elles dotent ainsi Wagah d'un moment où se déroule une action unique. Le lieu n'est plus seulement un point de contact sur la frontière, il est un lieu où est orchestré un rituel journalier autour de deux éléments emblématiques de la nation : l'armée qui la défend et le drapeau qui la représente.

Les discours sur l'origine de la cérémonie sont variés. Cependant, tous les interlocuteurs lui donnent une profondeur historique, liée à un grand moment des relations indo-pakistanaises. Pour certains, elle daterait de la partition en $1947^{10}$; selon l'office du tourisme du Punjab de 1949, elle aurait été instaurée au moment du cessez-le-feu sous l'égide de l'ONU permettant la création de la frontière et des Joint Check Points; enfin pour les officiers de la Border Security Force, elle aurait été instituée suite à la guerre indo-pakistanaise de 1965, en même temps que la création de leur unité spécialisée dans la surveillance de la frontière. Dans son organisation, elle est fortement liée au statut très spécifique du drapeau national indien dont l'usage est réglementé par un code, le Prevention of Insults to National Honour Act de 1971, amendé en $2003^{11}$. Le drapeau pakistanais, dont l'usage est beaucoup moins réglementé, intègre cependant les règles du drapeau indien à la frontière, où il ne peut pas apparaître comme inférieur. Par conséquent, les deux drapeaux sont descendus simultanément, dans une forme de concurrence symbolique où chaque nation doit rester toujours au même niveau que l'autre. En même temps, les deux forces de sécurité mettent en scène « la retraite » selon les codes du drill, le code hérité de l'armée britannique. Le geste doit être vif, voire violent et claquer aux ordres d'une voix forte et cinglante. De ce fait, le rite de descente du drapeau devient un théâtre pour exacerber le face-à-face entre les deux nations.

9. Cependant, ce discours d'intentions a été immédiatement remis en question par la guerre de Kargil planifiée à peine deux semaines après la déclaration commune par l'armée pakistanaise et entamée en mai 1999, dès la fonte des neiges.

10. "The show of anger and determination", Daily Times, Mardi 29 mai 2003.

11. Le drapeau indien est réglementé dès 1950 par le "Emblems and Names (Prevention of Improper Use) Act" qui est complété en 1971 par le "Prevention of Insults to National Honour Act". Le "Flag Code of India" applicable à partir du 26 janvier 2002 répond à la demande sociale d'usage individuel du drapeau, précédemment proscrite en dehors des fêtes nationales, suite à la pétition présentée par l'industriel Naveen Jindal à la Haute cour de Delhi. 


\section{Venir à Wagah Border pour vivre la nation et attester de la partition}

Si la cérémonie est ancienne de plusieurs dizaines d'années, elle est restée peu médiatisée avant les années 2000. Cependant, l'afflux croissant de spectateurs favorise l'émergence de nouvelles pratiques. À la descente des drapeaux est par exemple ajouté un long prologue patriotique au cours duquel les foules, incarnant les nations, investissent l'espace de la frontière.

La frontière, comme point de contact avec l'Autre, considéré parfois comme un adversaire voire un ennemi, permet de se mettre en scène. Ainsi, les touristes nationaux, qu'ils soient indiens ou pakistanais, vont à la fois observer et participer à la cérémonie. Cette double dimension est particulièrement visible dans le comportement adopté sur le moment et dans l'usage des images prises sur l'instant, puis montrées plus tard. Les visiteurs veulent à la fois être spectateurs et acteurs (Michel 2004 : 106). Wagah est un lieu qui se vit.

L'accès est donc ritualisé. Les visiteurs attendent sur la route goudronnée, devant les arches derrières les gradins, à la hauteur des monuments qui rappellent les massacres de $1947^{12}$. Du coté pakistanais, la porte de la liberté commémore les morts musulmans sur les chemins de l'exil, du coté indien, une stèle noire est dressée au milieu de la route à la mémoire des Punjabis massacrés durant la partition. Vers $18 \mathrm{~h}$, les femmes puis les hommes sont invités à pénétrer sur le site, selon un chemin précis. Les forces de sécurité leur font longer la frontière, pour pouvoir photographier la borne qui marque le « point 0 » et toucher les portes qui séparent les deux États. Ce parcours fait entrer les touristes par la scène et leur permet surtout de voir, à quelques mètres de là, le peuple voisin avant de prendre place dans les gradins ${ }^{13}$. Les Rangers pakistanais imposent à la population une tenue longue et la séparation des sexes dans les gradins, avec les hommes à droite et les femmes à gauche, au nom du respect des règles de l'Islam. Les forces de sécurité indiennes réservent, elles, un espace aux femmes et aux VIP, pour les mettre à distance des éventuels débordements des groupes de jeunes hommes, souvent exaltés par la cérémonie. En effet, l'attente n'est pas muette et calme, mais bruyante et démonstrative. Les deux États diffusent, à grands renforts de haut-parleurs, des musiques patriotiques. Au Pakistan, l'hymne national est associé à des psalmodies du Coran et tout particulièrement la Chahâda. En Inde, sont mélangés hymne et chants patriotiques, dont certains sont tirés de films populaires de Bollywood. Si les deux foules scandent « longue vie à l'Inde » ou « longue vie au Pakistan », les Indiens sont invités à danser devant les gradins, créant une ambiance de liesse généralisée. Mais surtout, certains individus sont appelés à jouer un rôle particulier. Ils vont pouvoir manipuler le drapeau national sous le contrôle des forces de sécurité. Des deux cotés de la frontière, ces individus deviennent, momentanément, des allégories de la nation.

12. Le Punjab était une région multiconfessionnelle qui fut partagée entre l'Inde et le Pakistan, selon des critères religieux. L'Ouest devenait musulman, tandis que l'Est devenait sikh et hindou. Les transferts de populations furent accompagnés de violences extrêmes qui ont accéléré les exodes contraints et le processus de recomposition religieuse.

13. Dans les nombreuses vidéos mises en ligne sur internet, il est intéressant de noter les zooms réguliers sur le public adverse. Certains aiment à s'attarder sur des visages comme pour humaniser une foule qui vocifère des slogans. 


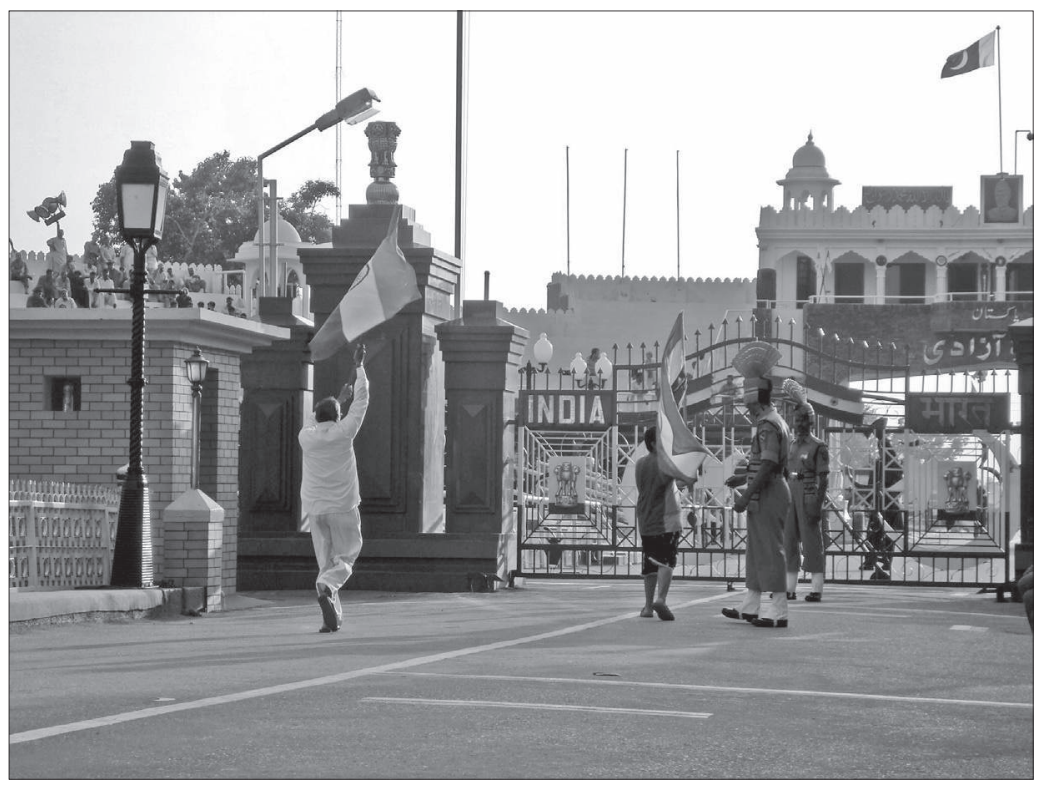

Course de drapeaux à Wagah Border (C) David Goeury

En Inde, un animateur appelle des spectateurs à brandir le drapeau national et à courir jusqu'à la frontière, sous les acclamations de la foule. Il choisit n'importe quel citoyen de passage pour démontrer la dimension démocratique de l'État. Les hommes, puis les femmes, se pressent pour bénéficier de ce moment de gloire, car en Inde, porter le drapeau ne se fait que dans des circonstances exceptionnelles, encadrées par l'État (Zins 2005) ${ }^{14}$. Les Indiens, choisis comme porte-étendards, ont le sentiment de vivre un moment exceptionnel, où devant des milliers de compatriotes, ils incarnent la nation, en portant son symbole, le drapeau, jusqu'aux limites terrestres de son territoire. Au Pakistan, tous les soirs, les deux mêmes hommes, vêtus de vert et de blanc, couleurs de la nation, paradent et exhortent la foule. Le plus âgé, Baba Mehar Din, vendeur de légumes, ancien soldat de 80 ans, résidant à Lahore, qui vient, chaque soir, depuis 40 ans (selon les journaux pakistanais), en scandant «Allah Akbar» (Dieu est grand) et «Pakistan Zindabad» (Longue vie au Pakistan), revendique son geste comme une participation à grandeur de la nation. Il est, d'ailleurs, devenu un mythe patriotique. Son engagement a été récompensé par le Président Musharraf, au nom de la nation, le 6 avril 2007, par la remise de 50000 Roupies et l'offre d'un voyage à la Mecque pour procéder à l'umra' .

14. Tout usage détourné est condamné. The Prevention of Insults to National Honour Act, section III, partie A, article v, 1971.

15. "Baba Mehar gets Rs 50000 for patriotism", Daily Times, Vendredi 6 avril 2007. L'umra est une visite à la Mecque qui peut se faire à tout moment à la différence du pèlerinage, elle ne fait pas partie des cinq piliers de l'islam et est souvent considérée comme supplémentaire ou préparatoire, elle efface les péchés de l'année. 


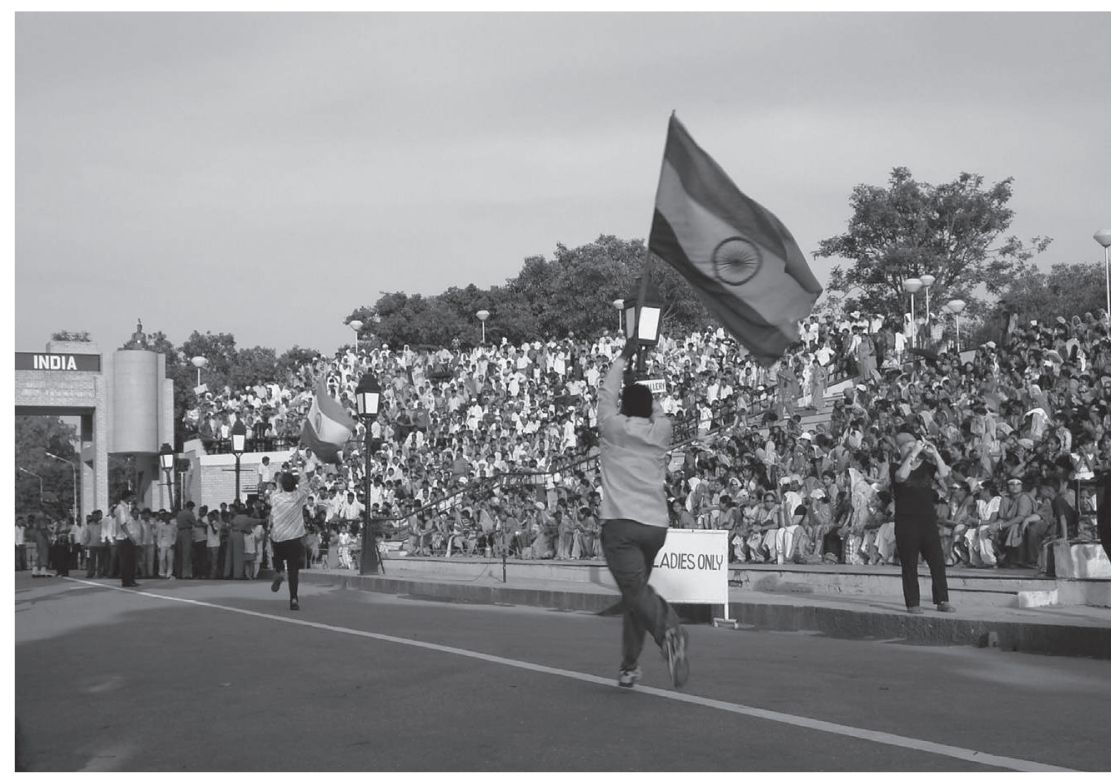

Course de drapeaux à Wagah Border (C) David Goeury

Les visiteurs nationaux sont mobilisés autour de symboles. D'un coté, le pays des purs s'affirme autour de la religion musulmane, alors que l'Inde exalte sa dimension démocratique et libre. La frontière et le point « 0 » deviennent le lieu témoin de la séparation entre l'Inde et le Pakistan. La partition y est attestée par tout individu, indien, pakistanais ou étranger, qui assiste à la cérémonie. L'affirmation de Mohammad Ali Jinnah « en 1947 nous étions une nation qui cherchait un pays, désormais nous sommes un pays qui cherche une nation » toujours au cœur du questionnement sur la nation pakistanaise, considérée comme inachevée (Jaffrelot 2002 : 7), trouve sa réponse à Wagah. Tous les symboles du Pakistan sont convoqués. Le portrait de Mohammad Ali Jinnah domine la porte de la liberté qui commémore la mort des milliers de réfugiés, victimes des violences interconfessionnelles en 1947. L'armée forte et inflexible garde la porte. L'Islam, ciment fédérateur, envahit l'espace sonore à travers les chants coraniques et s'incarne dans l'allégorie de Baba Mehar Din, drapé de vert, agitant le drapeau au croissant blanc.

De plus, les Pakistanais et Indiens construisent leurs identités nationales respectives en négatif de celle de l'autre. À l'exubérance et la démesure indienne, qui associent les musiques de films, les danses, la dimension bariolée de la foule et la course effrénée des porteurs d'étendards, comme autant de performances patriotiques individuelles qui témoignent de l'adhésion positive de chaque citoyen à « la plus grande démocratie du monde », les Pakistanais opposent la mesure du pays des Purs ${ }^{16}$. Pour les autorités pakistanaises qui cherchent à faire reconnaître la spécificité culturelle de leur nation à l'échelle mondiale, Wagah border devient l'exemple explicite de leur différence avec

16. Pour Maneesha Tikekar, la culture pakistanaise veut se construire comme une contre-culture indienne et une « anti-performing culture » (Tikekar 2004 : 330). 
l'Inde. Ainsi, tout groupe d'officiels étrangers de passage à Lahore est emmené à la cérémonie de la fermeture de la frontière, pour qu'ils puissent constater de leurs propres yeux que les deux nations, pakistanaise et indienne, sont clairement distinctes et surtout que les deux peuples ont des comportements opposés. Wagah leur permet de soutenir les discours sur l'impossible vie en commun entre Pakistanais et Indiens, de dénoncer le mouvement gandhien qui juge la partition comme le plus grand drame de l'Union indienne et enfin de revendiquer le rattachement du Cachemire au Pakistan (Jaffrelot $2000: 201)$.

Pour la population, Wagah Border devient alors l'espace où se vit la nation. Du coté indien, la cérémonie est rapidement devenue extrêmement populaire, faisant de Wagah une grande destination du tourisme national. Les transports collectifs se multiplient, attendant les clients à la sortie du Temple d'or sikh d'Amritsar. Les chauffeurs vantent l'incroyable de la cérémonie à renfort de photographies et surtout jouent sur la fibre nationaliste en arguant que c'est le lieu où tout citoyen indien qui se respecte doit être allé. Désormais, les professionnels du tourisme comparent le Taj Mahal d'Âgrâ et le Fort Rouge de New Delhi, les deux monuments nationaux, les plus visités, avec les deux principales attractions d'Amritsar. Ainsi, le Temple d'Or, chef d'œuvre d'architecture, est comparé au Taj Mahal. Cependant, le lieu de culte des Sikhs, toujours actif, fréquenté par des dizaines de milliers de pèlerins, est qualifié de «living monument» (monument vivant), alors que le tombeau moghol n'est que le fabuleux vestige d'une époque révolue. De même, Wagah border est présentée comme le lieu vivant de la nation indienne, alors que le Fort Rouge, où a été proclamée l'indépendance le 15 août 1947, n'est qu'un lieu de mémoire de la nation (Nora 1984). À la commémoration annuelle est opposée la possibilité de vivre le nationalisme face au «frère ennemi ». Des voyages spéciaux sont organisés pour les écoles et des groupes de jeunes hommes des grandes villes du Nord et du Sud viennent spécialement pour la fermeture de la frontière. Les étudiants et les jeunes urbains décrivent Wagah comme une expérience extrême, un contact direct et passionnel avec le nationalisme. Ils font de la frontière une destination à la mode où on doit être allé. Porter l'étendard et scander des slogans, permet de faire corps avec la nation, en longeant son enveloppe, la frontière avec le Pakistan (Equipe MIT 2002 : 197).

\section{La violence comme catharsis : un théâtre touristique}

L'augmentation croissante des visiteurs fait de Wagah border un véritable phénomène de société. Par conséquent, la cérémonie est devenue un espace de débat qui questionne ce qui relève du théâtre et du vécu des spectateurs. Deux points de vue s'opposent. D'une part, de nombreux observateurs, journalistes, universitaires et touristes internationaux interprètent la violence comme réelle. Pour ces «manipulateurs de symboles» ${ }^{17}$, la parade militaire avec ses gestes brutaux et intimidants, dont les fameux grands mouvements de jambes ressemblant à de terribles coups de pieds, est bien plus que du théâtre. Ainsi, dans son livre Across the Wagah an Indian Sejourn in Pakistan, l'universitaire indienne, Maneesha Tikekar décrit cette scène comme un affrontement dramatique où la haine des regards, la violence des coups de pieds en l'air sont autant d'appels à la violence.

17. Nous utilisons l'expression de Robert Reich pour designer ces acteurs mondialisés pouvant largement diffuser leurs interprétations et créer des représentations du Monde. 
Pour elle, exhiber le patriotisme avec autant d'animosité envers son voisin, et tant de plaisir à le faire, est un jeu dangereux. Elle conclut son analyse en se demandant s'il existe ailleurs dans le monde une descente de drapeau aussi théâtrale et aussi excessive de patriotisme (Tikekar 2004 : 92). De même, l'artiste et militant pakistanais Mian Ijazul-Hassan voit en la violence pathétique de la cérémonie de fermeture de la frontière de Wagah qui recommence inlassablement chaque soir, l'image du conflit indo-pakistanais ${ }^{18}$. Enfin, dans un texte illustré de photographies de la cérémonie et intitulé «The Wagah Syndrome ", l'universitaire Willem Van Schendel fait de la cérémonie le modèle type de l'ultranationalisme exubérant dont la débauche de violences symboliques annonce des violences physiques vis-à-vis des États voisins et à l'encontre des communautés minoritaires (Van Schendel 2007 : 44). Si l'élite intellectuelle indienne et pakistanaise condamne la cérémonie, d'autres voix plus anonymes distinguent théâtralisation de l'agressivité et violence ${ }^{19}$. En effet, sur place, nombreux sont les Pakistanais et les Indiens qui n'y voient qu'un show. Les Punjabis, habitués à y conduire leurs amis venus en visite, présentent Wagah comme une attraction, sans pourtant exclure sa dimension civique. Ainsi sur le site internet de mise en ligne de vidéos personnelles Dailymotion, un Pakistanais a décidé d'intégrer un prologue à son montage d'images de la cérémonie en disant «n'y voyez rien de politique ou de guerrier et si vous n'êtes pas d'accord, contactez-moi pour en discuter $\gg$.

Le 20 octobre 2002, ce débat est devenu institutionnel, suite à l'affrontement entre un soldat pakistanais et un soldat indien qui se sont menacés de leurs armes à feu, heureusement déchargées durant la cérémonie; ils ont du être séparés par les officiers alors que les spectateurs des deux cotés multipliaient les insultes ${ }^{20}$. Pendant plus de deux ans, les deux administrations militaires ont débattu du degré de violence des gestes hérités du drill. À la fin du mois de mars 2004, l'Inde a demandé officiellement de réduire l'agressivité des gestes et surtout l'ampleur des mouvements de jambes pour qu'ils apparaissent comme moins brutaux et ressemblent moins à un simulacre de combat. À Lahore, le commandement des Border Security Forces indiennes et des Rangers pakistanais ont décidé de réorienter la cérémonie vers des postures moins agressives en demandant aux soldats de ne faire aucune provocation et bien au contraire de faire preuve de respect mutuel. Cependant après l'accord de principe, les deux parties ont convenu qu'il fallait d'abord déterminer la forme du nouveau rituel avant de changer ${ }^{21}$. Or, en novembre 2006, le lieutenant général Hussain Mehdi, directeur général des Rangers pakistanais du Punjab, a déclaré qu'il ne ferait pas modifier la gestuelle des soldats, alors que les Indiens avaient procédé à de légères modifications pour adoucir les mouvements. Pour lui, la cérémonie de la "retraite » héritée du drill doit perdurer dans sa forme originelle, car elle est à la fois un élément identitaire de la population pakistanaise et surtout, désormais, une attraction touristique. Il conclut que la prétendue violence de la cérémonie a un très

18. Ijaz-ul-Hassan M. , "The way it was: Other side of nationalism”, Daily Times, 11 juin 2003.

19. Ces anonymes ne bénéficient pas des mêmes médias pour diffuser leur point de vue.

20. AFP, "Ceremonial guard at India-Pakistan border ends in brawl”, Daily Times, 22 octobre 2002.

21. Gilani I., "Flag lowering at Wagah border : Pakistan, India decide to 'reorient' ceremony", Daily Times, 7 avril 2004. 
faible impact sur les relations bilatérales indo-pakistanaises ${ }^{22}$. Le débat se poursuit et les autorités pakistanaises, à l'image du lieutenant-colonel Tariq Janjua, considèrent même que la diminution de la dramaturgie gâcherait le spectacle alors que la foule vient pour la dimension démesurée du drill et non pour une simple parade s'achevant par la descente du drapeau et la fermeture de la frontière ${ }^{23}$. Les plus hauts dignitaires de l'État pakistanais, le Général major Javid Zia et le ministre de l'Information Tariq Azim, réaffirment cette idée, lors des rencontres avec leurs homologues indiens à New Delhi ${ }^{24}$. L'afflux croissant de touristes nationaux et internationaux justifie, alors, l'agressivité des soldats comme un élément du show.

L'attrait du spectacle ne doit pas écarter la dimension idéologique du débat. Le refus pakistanais, s'inscrit dans la permanence du mythe colonial des races dites « martiales ». De cette théorie britannique a émergé l'idée de la supériorité du soldat musulman carnivore sur le soldat hindou végétarien ${ }^{25}$. Ce mythe reste très présent dans les esprits de la population et des officiers pakistanais et ce malgré plusieurs défaites face aux Indiens ${ }^{26}$. L'idée d'agressivité et de force des soldats musulmans est constitutive de l'ethos de l'armée pakistanaise. Elle est utilisée pour justifier la résistance du Pakistan à l'Inde, malgré le différentiel démographique entre les deux États (166 millions d'habitants au Pakistan contre 1142 millions en Inde) ${ }^{27}$. Les Rangers de Wagah border grands, musculeux, au regard furieux, surnommés avec fierté « les fils de lions », doivent incarner la prétendue supériorité militaire devant l'armée indienne, pas tant pour impressionner les soldats indiens que pour rassurer le public pakistanais (Jaffrelot 2000 : 204-205). Le rituel doit donc conserver son agressivité.

Cependant, sur place, les hommes qui manifestent le plus de ferveur patriotique et qui s'enflamment en hurlant des slogans, concluent toute discussion sur le sujet en disant que les tensions entre l'Inde et le Pakistan sont le fruit des gouvernements et surtout, pour les Indiens, de l'absence de démocratie au Pakistan. Wagah draine un public de plus en plus nombreux sans qu'il y ait de violences physiques, alors que le Pakistan et l'Inde sont régulièrement le lieu de violences communautaires. Les spectateurs de la cérémonie de la fermeture de la frontière, même lorsqu'ils exultent verbalement, restent pacifiques. La frontière n'est pas un lieu de conflit avec l'Autre, mais de confrontation à l'Autre pour construire son identité. La théâtralisation désamorce le conflit, par la reconnaissance de l'Autre dans sa différence (Sémelin 2005 : 444-445).

22. Khan S., "Rangers won't tone down drill”, Daily Times, 13 novembre 2006.

23. "Crowds left cold as India leaves Wagah dramatic to Pakistan”, Reuters, 13 janvier 2007.

24. "Troops to keep up aggressive ritual at Wagah", AFP, 18 février 2007.

25. Cette théorie permettait de justifier l'autorité des empires musulmans depuis le $12^{\mathrm{e}}$ siècle alors que les hindous étaient majoritaires.

26. La dernière défaite durant la guerre de Kargil en 1999 n’a jamais été officiellement reconnue.

27. Il est intéressant de noter que le service de recrutement de l'armée pakistanaise met en avant, sur son site internet, l'invincibilité des soldats alors que l'armée indienne insiste surtout sur la fraternité et le courage. 


\section{Les 14 et 15 août : cérémonie et contre-cérémonie à Wagah}

Les 14 et 15 août, jours des fêtes nationales respectivement du Pakistan et de l'Union indienne, sont des moments forts à Wagah. Le nombre des visiteurs bat des records. La levée du drapeau pakistanais à la frontière le 14 août est le symbole de la naissance du Pakistan, séparé de l'Union indienne. Wagah Border est un des lieux où « l'on doit être » pour fêter l'indépendance comme Lahore pour les Pakistanais et le Fort Rouge à New Delhi pour les Indiens.

Wagah est, aussi, le lieu d'une autre cérémonie annuelle dont l'objectif est tout autre. Depuis le 14 août 1996, pour la 50 année de l'indépendance du Pakistan et de l'Inde, Kuldip Nayar, l'éditorialiste susmentionné, a décidé d'allumer des chandelles à minuit (Nayar 2003 : 208). Les chandelles restent allumées, à la frontière, toute la nuit, pour assurer une continuité symbolique entre les deux déclarations successives d'indépendance du Pakistan le 14 et de l'Union indienne le 15. À cette manifestation pour la paix et la réconciliation, ne participaient qu'une douzaine de personnes lors de la première expérience, alors qu'aujourd'hui, elle mobilise des centaines de milliers de personnes. La candlelight vigil (veillée aux chandelles) est désormais devenue un moment fort. Des associations, de part et d'autre de la frontière, de plus en plus nombreuses rallient le mouvement comme symbole de la paix possible entre l'Inde et le Pakistan. Elles associent aux chandelles des concerts associant musique moderne et de danse traditionnelle punjabie. Les participants scandent «Hindu-Pak Dosti Zindabad» (Longue vie à l'amitié indopakistanaise). Ils appellent à la fin de la politique des visas pour favoriser les liens directs entre les populations, pour en finir avec les affrontements par gouvernements interposés. Ce mouvement est fortement critiqué par les partisans indiens d'une politique dure visà-vis du Pakistan. Ils décrivent les porteurs de chandelles comme des doux rêveurs face à la violence et la dangerosité du Pakistan, pourvoyeur de guerre et de terrorisme. De même, les forces de sécurité des deux pays hésitent à laisser la population librement accéder au «point 0 ». Régulièrement, les gouvernements empêchent la manifestation ou en diminuent la portée symbolique. Par exemple, en 2004, si les Pakistanais sont autorisés à se rendre à la cérémonie de lever du drapeau le matin du 14 août, les autorités bloquent, dans la banlieue de Lahore, les individus désireux de gagner la frontière pour la veillée aux chandelles du soir, prétextant une menace terroriste islamiste. De son coté, la Border Security Force indienne préfère retenir les manifestants indiens à l'entrée de la zone douanière. Et même si certains militants pacifistes arrivent à passer les barrages, le Pakistan fait diffuser par les hauts-parleurs des chants coraniques pour couvrir leurs slogans prônant l'amitié entre l'Inde et le Pakistan ${ }^{28}$.

Pour le soixantième anniversaire de l'indépendance, 2007 devait être un moment exceptionnel. Deux associations « Routes 2 Roots $^{29}$ » et « Friends without Borders ${ }^{30} »$, qui

28. Ahmed S., Khan S., « Lahore the place to be on Aug 14 », Daily Times, 15 août 2004.

29. Cette association indienne basée à Delhi disposant d'une antenne au Pakistan est dirigée par l'indienne Amita Gupta et la pakistanaise Tina Vachani. Elle est soutenue par de nombreuses femmes qui veulent réconcilier les États du Sud asiatique et surtout le Pakistan et l'Inde comme l'ont été la RDA et la RFA. Elles organisent de multiples actions culturelles, des manifestations communes régulières et militent pour la création d'une zone économique de libre-échange entre les deux pays (voir http://www.routes2roots. com). 
multiplient les liens entre les populations des deux États, envisagent un immense concert « Dil se Dil (Heart to heart) : Independance Day Friendship Celebration » commun, de part et d'autre de la frontière. Il devait commencer le 14 août à $23 \mathrm{~h}$ et s'achever le 15 à $12 \mathrm{~h} 30$, pour faire vivre symboliquement la continuité entre les deux déclarations d'indépendance. Ce concert géant, financé par des multinationales comme Nokia, devait être retransmis en direct sur la télévision indienne et pakistanaise. Des stars du Bollywood, des joueurs de cricket et des chanteurs étaient attendus pour ce moment exceptionnel. L'association « Routes 2 Roots » voulait que la cérémonie montre qu'Indiens et Pakistanais participent d'une même humanité, dansent sur les mêmes chansons, idéalisent les mêmes stars et pratiquent les mêmes sports. Cependant, au dernier moment, les autorités locales ont décidé d'interdire l'accès nocturne à la zone frontière pour des raisons de sécurité ${ }^{31}$, tenaillées par la peur des mouvements de foule ${ }^{32}$. Le projet a donc été annulé.

Kuldip Nayar n'hésite pas à opposer la démarche des deux cérémonies médiatiques de Wagah que sont la descente du drapeau et la veillée aux chandelles. Pour lui, la première est étroitement encadrée par les institutions, dont l'armée, en évitant tout contact direct entre les populations, alors que la seconde appelle à la réconciliation directe entre les individus ${ }^{33}$. Cette opposition est en fait beaucoup plus profonde et révèle deux courants qui animent le sous-continent depuis 1947. En effet, la société reste divisée entre les partisans du rêve de la réconciliation et les partisans de la théorie des deux nations (Jaffrelot $2000: 231$ ). À la cérémonie nationaliste orchestrée par l'armée et l'État, la « candlelight vigil » oppose une logique transnationale qui dépasse la politique des États et s'appuie sur une société civile mondiale, soutenue par des ONG et des multinationales. La «candlelight vigil » nie la frontière en s'appuyant sur des valeurs mondiales présentées comme plus légitimes que celles de la nation. De ce fait, elle n'est pas seulement jugée comme une manifestation de doux rêveurs, mais comme une force subversive à même de remettre en question les constructions politiques nationales. L'argument du mouvement de foule franchissant la frontière sans passer par le filtre institutionnel des visas n'est pas un alibi fallacieux pour interdire le concert, mais traduit bel et bien la peur d'un acte symbolique fort qui serait médiatisé à l'échelle mondiale, remettant en question les logiques gouvernementales. Par conséquent, la manifestation est régulièrement entravée par les autorités indiennes et pakistanaises qui aujourd'hui s'abritent derrière le risque terroriste, dont la dimension imprévisible permet de justifier l'interdiction des grandes manifestations publiques.

Il faut noter que cette contre-cérémonie ne diminue pas l'aura de la descente des drapeaux, bien au contraire, elle la renforce. Les deux événements ont un effet cumulatif générant une aura toujours plus grande du poste-frontière et attirant un public de plus en plus nombreux.

30. Cette association basée aux États-Unis organise un vaste échange de courriers entre les enfants pakistanais et indiens pour favoriser des contacts directs entre les nouvelles générations. http://www.friendswithoutborders.org

31. Manan A., “Thousands celebrate at Wagah”, Daily times, 15 août 2007.

32. Jaideep Sarin, “Gala India-Pakistan concert at Wagah called off”, IANS, 14 août 2007.

33. Http://kuldipnayar.com/Express/10August2006.htm 


\section{Le tourisme : une première étape vers la normalisation des relations indo- pakistanaises}

La résonance nationale de Wagah Border ne doit pas occulter les enjeux régionaux. En effet, le projet a été porté en premier par l'État du Punjab et surtout par l'élite économique et administrative de la ville d'Amritsar dont de nombreux membres de la communauté sikhe (Foucher 1991 : 332). Cette minorité religieuse, concentrée dans le Punjab, a souffert de la partition qui l'a obligée à quitter le Punjab pakistanais et l'a coupée de lieux de cultes comme le lieu de naissance du guru Nanak. Après avoir réclamé le fractionnement de l'État du Punjab pour disposer d'un État sikh plus autonome, à la fin des années 1970, les ultranationalistes sikhs sont entrés en guerre avec le gouvernement fédéral de New Delhi pour créer un État indépendant, le Khalistan ${ }^{34}$. Battus, aujourd'hui, ils laissent la place à un nouveau mouvement sikh désireux de s'ouvrir sur le Pakistan. Il veut redonner au Punjab sa dimension d'espace charnière, et ainsi relancer le développement économique de la région, mis à mal par les années de conflits.

Dans ce nouveau contexte, le département du tourisme d'Amritsar désire jouer un rôle-clef et insiste sur les multiples dimensions de la cérémonie. Si effectivement, la médiatisation de la cérémonie permet d'augmenter la fréquentation touristique de la région et surtout le nombre de nuitées passées dans la ville, en mettant en scène une activité nocturne qui s'achève à $19 \mathrm{~h} 30$ et qui oblige, ainsi, les touristes à dormir à Amritsar, elle a aussi des objectifs politiques. L'administration revendique la dimension nationaliste de la cérémonie. Elle la présente comme un moment d'éducation à la citoyenneté, avec l'exaltation des symboles nationaux, et de civisme, par le respect des institutions et du public pakistanais. L'animateur indien s'assure que les éventuelles insultes ou slogans hostiles soient couverts par la voix de la foule scandant « longue vie à l'Inde». Alors, la cérémonie joue un rôle fondamental dans la politique nouvelle de réconciliation entre l'Union indienne et le Pakistan. Les responsables du département du tourisme espèrent modifier en profondeur la vision qu'ont les Indiens du Pakistan, par l'accueil d'un public de plus en plus diversifié, venant de toute l'Inde et surtout du Sud ${ }^{35}$. Ils veulent substituer à la représentation de la frontière-front d'où émergeraient régulièrement la guerre et le terrorisme, celle d'une frontière contrôlée où deux peuples proches et respectables peuvent se rencontrer. De ce fait, pour ces Punjabis, le tourisme de masse à Wagah est essentiel à la construction d'un premier dialogue avec le Pakistan, en permettant à la population indienne d'entrer, même de manière lointaine et extrêmement contrôlée, en contact avec la population pakistanaise et ainsi de la dédiaboliser. Ils insistent sur la dimension binationale de la cérémonie et sur la coordination des deux forces armées, prouvant qu'Indiens et Pakistanais peuvent travailler ensemble quotidiennement et sans heurts. Nombreux sont ceux qui concluent sur le fait que, la nuit venue, les soldats ne respecteraient pas la frontière et vont manger et boire ensemble. Ils espèrent ainsi que l'opinion publique nationale de l'Inde devienne plus favorable à la réouverture progressive

34. L'aventure se termine tragiquement par la prise d'assaut par l'armée du Temple d'or et l'assassinat quelques mois plus tard d'Indira Gandhi par ses gardes du corps sikhs en 1984 (Jaffrelot 2006 : 318).

35. Les officiels et les habitants d'Amritsar insistent sur le fait que la cérémonie soit, désormais, extrêmement populaire à l'échelle nationale. Ils mettent en avant la venue croissante d'Indiens vivant dans le Sud de la péninsule, sans pour autant être capables de donner des éléments statistiques. 
de la frontière à un trafic de marchandises plus intense. Certains avancent même l'espoir de mettre en place progressivement une zone de libre-échange avec le Pakistan, idée encore assez impopulaire à l'échelle nationale ${ }^{36}$.

Le $1^{\mathrm{er}}$ novembre 2007, une première étape a été franchie avec l'autorisation donnée aux camions préalablement enregistrés de traverser la frontière avec pour objectif d'atteindre un trafic de 200 camions par jour. L'État du Punjab et la ville d'Amritsar envisagent désormais la création d'une vaste zone commerciale qui accueillerait les camions le jour et les touristes le soir. Parallèlement, pour accélérer les échanges et la libre circulation des individus, l'État du Punjab espère l'implantation d'un consulat du Pakistan à Amritsar, habilité à délivrer des visas pour la population et surtout pour les pèlerins sikhs. La cérémonie est aujourd'hui présentée comme une première étape de la reconnaissance internationale de deux nations qui peuvent dépasser leurs conflits antérieurs et devenir des partenaires économiques et politiques. Certains décideurs punjabis espèrent la généralisation des partenariats internationaux dans le cadre d'une nouvelle association d'États, comme la SAFTA (South Asia Free Trade Association) créée en 2004, et ainsi recréer une aire de développement économique internationale transfrontalière, sur les vestiges du Punjab d'avant 1947, sans remettre en question l'idée des deux nations (Jaffrelot $2000: 232$ ).

\section{Conclusion}

Wagah Border est un haut lieu des relations indo-pakistanaises qui porte en lui à la fois la mémoire de la violence des massacres communautaires de 1947 et le quotidien des conflits frontaliers. Cette dimension symbolique forte est renforcée par la cérémonie quotidienne de descente des drapeaux. Gouvernements fédéraux, gouvernements régionaux, armées, partis politiques, intellectuels, associations transnationales, investissent ce lieu pour légitimer des discours sur les relations indo-pakistanaises. Malgré leurs points de vue divergents, voire opposés, ils dotent Wagah Border d'une résonance grandissante et renforcent son attractivité touristique. Or, la mise en tourisme de la frontière transforme la symbolique de la cérémonie. L'agressivité des soldats devient une composition d'acteurs, le nationalisme, un jeu. La théâtralisation rapproche la cérémonie du show et l'écarte de la guerre. Ceci est renforcé par la dimension journalière, donc répétitive, de cet affrontement symbolique entre deux armées, sans vaincu et sans vainqueur, sous les acclamations d'une foule en liesse. Dès lors, le face-à-face des touristes indiens et pakistanais permet d'attester de la partition et de légitimer les institutions incarnées par les deux armées et les deux drapeaux. Il est une première étape de la reconnaissance de l'Autre, avec ses spécificités. Cette étape est indispensable à la normalisation des relations internationales. Par conséquent, ce tourisme de masse, même s'il se fait autour des symboles de la nation, est un élément de pacification du lieu. Il permet de convertir un espace de conflit en un espace de conciliation. Il dépasse les débats idéologiques et instaure les conditions d'une première rencontre, certes limitée, mais réelle. De plus, il prépare le passage d'une gestion purement politique de la frontière à une gestion plus économique, en générant de nouveaux flux et de nouvelles activités (Brunel 2006 : 257). Enfin, il permet de concilier enjeux nationaux et développement régional, surtout dans une démocratie où la prise de décision passe par l'adhésion des masses et la sensibilisation des décideurs.

36. Entretiens avec 1'auteur, août 2007. 


\section{Références citées}

Brunel, Sylvie, 2006. La planète disneylandisée. Chroniques d'un tour du monde. Paris : Éditions Sciences Humaines.

ÉQUIPE MIT,

2002. Tourismes 1. Lieux communs. Paris : Belin

2005. Tourismes 2. Moments de lieux. Paris : Belin.

Foucher, Michel, 1991. Fronts et frontières. Un tour du monde géopolitique. Paris : Fayard.

Gelbman, Alon, 2008. "BorderTourism in Israel: Conflict, Peace, Fear and Hope", Tourism Geographies, 10 (2), pp. 193-213.

Halbwachs, Maurice, 1950. La mémoire collective. Paris : PUF.

JAFFRELOT, Christophe,

2000. Le Pakistan. Paris : Fayard.

2002. "Pakistan searching for its Identity", in Jaffrelot C. (dir.), Pakistan Nationalism without a Nation?, pp. 7-51. New Delhi: Manohar.

2006. L'Inde contemporaine, de 1950 à nos jours. Paris : Fayard.

Michel, Franck, 2004. Désirs d'ailleurs. Essai d'anthropologie des voyages. Québec : Presses de l'université de Laval.

NAYAR, Kuldip, 2003. Wall at Wagah: India-Pakistan Relation. New Delhi: Gyan Pub. House.

Nora, Pierre (dir.), 1984. Les lieux de mémoire. I. La République. Paris : Gallimard.

SÉmelin, Jacques, 2005. Purifier et détruire. Les usages politiques des massacres et génocides. Paris : Seuil.

TikeKar, Maneeshar, 2004. Across the Wagah, an Indian Sejourn in Pakistan. New Delhi/New Jersey: Promilla and co./Publishers bibliophile South Asia.

VAN Schendel, Willem, 2007. "The Wagah Syndrome: Territorial Roots of Contemporary Violence in South Asia”, in Basu, Amrita et Srirupa Roy (eds), Violence and democracy in India, pp. 36-82. Calcutta, New York, Londres: Seagull Books.

ZINS, Max-Jean, 2005. «Rites publics et deuil patriotique. Les funérailles de la guerre indo-pakistanaise de $1999 »$, Archives des sciences sociales des religions, 131 (2005) - Varia, (En ligne), mis en ligne le 30 juin 2008. URL : http://assr.revues.org/document $3258 . h t m l$ 